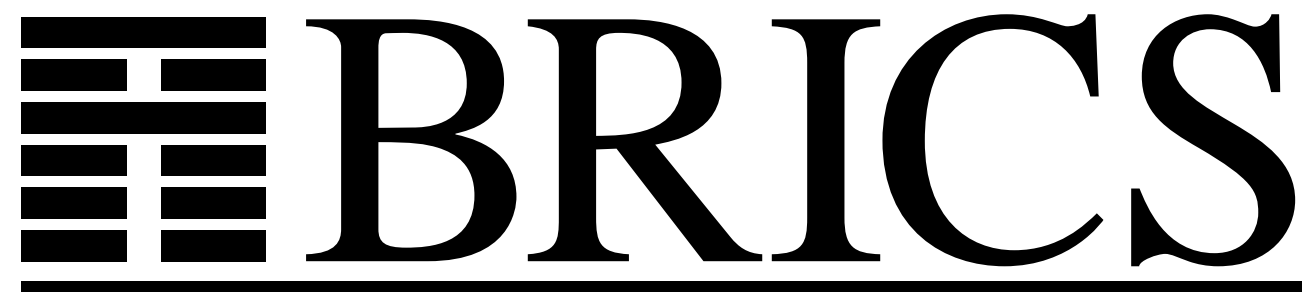

Basic Research in Computer Science

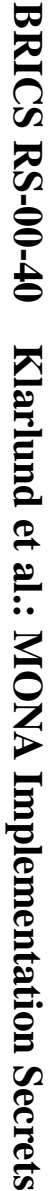

\title{
MONA Implementation Secrets
}

Nils Klarlund

Anders Møller

Michael I. Schwartzbach 
Copyright (c) 2000, Nils Klarlund \& Anders Møller \& Michael I.

Schwartzbach.

BRICS, Department of Computer Science

University of Aarhus. All rights reserved.

Reproduction of all or part of this work is permitted for educational or research use on condition that this copyright notice is included in any copy.

See back inner page for a list of recent BRICS Report Series publications. Copies may be obtained by contacting:

\author{
BRICS \\ Department of Computer Science \\ University of Aarhus \\ Ny Munkegade, building 540 \\ DK-8000 Aarhus C \\ Denmark \\ Telephone: +4589423360 \\ Telefax: $\quad+4589423255$ \\ Internet: BRICS@brics.dk
}

BRICS publications are in general accessible through the World Wide Web and anonymous FTP through these URLs:

http://www.brics.dk

ftp: / / ftp.brics.dk

This document in subdirectory RS / $00 / 40$ / 


\title{
MONA Implementation Secrets
}

\author{
Nils Klarlund \\ AT\&T Labs Research \\ Florham Park, NJ \\ klarlund@research.att.com
}

\author{
Anders Møller \& Michael I. Schwartzbach \\ BRICS \\ Department of Computer Science \\ University of Aarhus, Denmark \\ $\{$ amoeller,mis\}@brics.dk
}

\begin{abstract}
The Mona tool provides an implementation of automaton-based decision procedures for the logics WS1S and WS2S. It has been used for numerous applications, and it is remarkably efficient in practice, even though it faces a theoretically non-elementary worst-case complexity. The implementation has matured over a period of six years. Compared to the first naive version, the present tool is faster by several orders of magnitude. This speedup is obtained from many different contributions working on all levels of the compilation and execution of formulas. We present an overview of MONA and a selection of implementation "secrets" that have been discovered and tested over the years, including formula reductions, DAGification, guided tree automata, three-valued logic, eager minimization, BDD-based automata representations, and cache-conscious data structures. We describe these techniques and quantify their respective effects by experimenting with separate versions of the MONA tool that in turn omit each of them.
\end{abstract}

\section{Introduction}

Mona $[21,30,38,26]$ is an implementation of decision procedures for the logics WS1S and WS2S (Weak monadic Second-order theory of 1 or 2 Successors) [48]. They have long been known to be decidable $[12,18]$, but with a non-elementary lower bound [37]. For many years it was assumed that this discouraging complexity precluded any useful implementations.

MONA has been developed at BRICS since 1994, when our initial attempt at automatic pointer analysis through automata calculations took four hours to complete. Today MonA has matured into an efficient and popular tool on 
which the same analysis is performed in a couple of seconds. Through those years, many different approaches have been tried out, and a good number of implementation "secrets" have been discovered. This paper describes the most important tricks we have learned, and it tries to quantify their relative merits on a number of benchmark formulas.

Of course, the resulting tool still has a non-elementary worst-case complexity. Perhaps surprisingly, this complexity also contributes to successful applications, since it is provably linked to the succinctness of the logics. If we want to describe a particular regular set, then a WS1S formula may be non-elementarily more succinct that a regular expression or a transition table.

The niche for MonA applications contains those structures that are too large and complicated to describe by other means, yet not so large as to require infeasible computations. Happily, many interesting projects fit into this niche, including hardware verification $[4,1]$, pointer analysis [23, 17], controller synthesis [44, 22], natural languages [39], parsing tools [14], software design descriptions [29], Presburger arithmetic [45], and verification of concurrent systems $[32,31,24,42,46]$.

There are a number of tools resembling MONA. Independently of the MONA project, the first implementation of automata represented with BDDs was that of Gupta and Fischer from 1993 [20]. However, they used "linear inductive functions" instead of the automaton-logic connection. MoSEL (see http://sunshine. cs.uni-dortmund.de/projects/mosel/) implements the automaton based decision procedure for M2L-Str using BDDs like MonA. In [25], MoSEL is described and compared with Mona 0.2, which provided inspiration for the MoSEL project. Apparently, there have been only few applications of MoSEL. AMoRE [35] (see http://www.informatik.uni-kiel.de/inf/Thomas/amore.html) is a library of automata theoretic algorithms, resembling those used in MonA. AMoRE also provides functionality for regular expressions and monoids, but is not tied to the automaton-logic connection. Glenn and Gasarch [19] have in 1997apparently independently of MONA and MOSEL - implemented a decision procedure for WS1S, basically as the one in MONA, but without using BDDs or other sophisticated techniques. The SHASTA tool from 1998 is based upon the same ideas as MonA. It is used as an engine for Presburger Arithmetic [45].

Furthermore, Mona has provided the foundation of or been integrated into a range of other tools: FIDO [34], LISA [2], DCVALID [42], FMONA [9, 8], STTOOLS [43], PEN [40], PAX [5], PVS [41], and ISABELLE [3].

\section{The Automaton-Logic Connection}

Being a variation of first-order logic, WS1S is a formalism with quantifiers and boolean connectives. First-order terms denote natural numbers, which can be compared and subjected to addition with constants. Also, WS1S allows secondorder terms, which are interpreted as finite sets of numbers. The actual MonA syntax is a rich notation with constructs such as set constants, predicates and macros, modulo operations, and let-bindings. If all such syntactic sugar is peeled 
off, the formulas are "flattened" (so that there are no nested terms), and firstorder terms are encoded as second-order terms, the logic reduces to a simple "core" language ( $X$ ranges over a set of second-order variables):

$$
\begin{array}{ll|l|l}
\phi \quad:=\sim \phi^{\prime} & \phi^{\prime} \& \phi^{\prime \prime} & \text { ex2 } X_{i}: \phi^{\prime} \\
& X_{i} \operatorname{sub} X_{j} & X_{i}=X_{j} \backslash X_{k} & X_{i}=X_{j}+1
\end{array}
$$

Given a fixed main formula $\phi_{0}$, we define its semantics inductively relative to a string $w$ over the alphabet $\mathbb{B}^{k}$, where $\mathbb{B}=\{0,1\}$ and $k$ is the number of variables in $\phi_{0}$. We assume every variable of $\phi_{0}$ is assigned a unique index in the range $1,2, . ., k$, and that $X_{i}$ denotes the variable with index $i$. The string $w$ determines an interpretation $w\left(X_{i}\right)$ of $X_{i}$ defined as the finite set $\left\{j \mid\right.$ the $j$ th bit in the $X_{i}$-track is 1$\}$.

The semantics of a formula $\phi$ in the core language can now be defined inductively relative to an interpretation $w$. We use the notation $w \vDash \phi$ (which is read: $w$ satisfies $\phi$ ) if the interpretation defined by $w$ makes $\phi$ true:

$$
\begin{aligned}
& w \vDash \sim \phi^{\prime} \quad \text { iff } \quad w \not \models \phi^{\prime} \\
& w \vDash \phi^{\prime} \& \phi^{\prime \prime} \quad \text { iff } \quad w \vDash \phi^{\prime} \text { and } w \vDash \phi^{\prime \prime} \\
& w \vDash \operatorname{ex} 2 X_{i}: \phi^{\prime} \quad \text { iff } \quad \exists \text { finite } M \subseteq \mathbb{N}: w\left[X_{i} \mapsto M\right] \vDash \phi^{\prime} \\
& w \vDash X_{i} \operatorname{sub} X_{j} \quad \text { iff } \quad w\left(X_{i}\right) \subseteq w\left(X_{j}\right) \\
& w \vDash X_{i}=X_{j} \backslash X_{k} \quad \text { iff } \quad w\left(X_{i}\right)=w\left(X_{j}\right) \backslash w\left(X_{k}\right) \\
& w \vDash X_{i}=X_{j}+1 \quad \text { iff } \quad w\left(X_{i}\right)=\left\{j+1 \mid j \in w\left(X_{j}\right)\right\}
\end{aligned}
$$

The notation $w\left[X_{i} \mapsto M\right]$ is used for the shortest string that interprets all variables $X_{j}$ where $j \neq i$ as $w$ does, but interprets $X_{i}$ as $M$.

The language $\mathcal{L}(\phi)$ of a formula $\phi$ can be defined as the set of satisfying strings: $\mathcal{L}(\phi)=\{w \mid w \vDash \phi\}$. By induction in the formula, we can now construct a minimal deterministic finite-state automaton (DFA) $A$ such that $\mathcal{L}(A)=\mathcal{L}(\phi)$, where $\mathcal{L}(A)$ is the language recognized by $A$.

For the atomic formulas, we show just one example: the automaton for the formula $\phi=X_{i}$ sub $X_{j}$ in the case where $i=1$ and $j=2$. The automaton must recognize the language

$$
\begin{aligned}
\mathcal{L}\left(X_{1} \text { sub } X_{2}\right)=\left\{w \in\left(\mathbb{B}^{k}\right)^{*} \mid\right. & \text { for all letters in } w: \text { if the first component is } \\
& 1, \text { then so is the second }\}
\end{aligned}
$$

Such an automaton is:

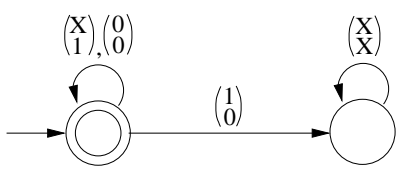

The other atomic formulas are treated similarly. The composite formulas are translated as follows:

$\phi=\sim \phi^{\prime} \quad$ Negation of a formula corresponds to automaton complementation. In MonA, this is implemented trivially by flipping accepting and rejecting states. 
$\phi=\phi^{\prime} \& \phi^{\prime \prime} \quad$ Conjunction corresponds to language intersection. In MoNA, this is implemented with a standard automaton product construction generating only the reachable product states. The resulting automaton is minimized.

$\phi=\operatorname{ex} 2 X_{i}: \phi^{\prime} \quad$ Existential quantification corresponds to a simple quotient operation followed by a projection operation. The quotient operation takes care of the problem that the only strings satisfying $\phi^{\prime}$ may be longer than those satisfying ex2 $X_{i}: \phi^{\prime}$. The projection operation removes the "track" belonging to $X_{i}$, resulting in a nondeterministic automaton, which is subsequently determinized using the subset construction operation, and finally minimized.

This presentation is a simplified version of the procedure actually used in MONA. For more detail, see the MONA User Manual [30].

When the minimal automaton $A_{0}$ corresponding to $\phi_{0}$ has been constructed, validity of $\phi_{0}$ can be checked simply by observing whether $A_{0}$ is the one-state automaton accepting everything. If $\phi_{0}$ is not valid, a (minimal) counter-example can be constructed by finding a (minimal) path in $A_{0}$ from the initial state to a non-accepting state.

WS2S is the generalization of WS1S from linear- to binary-tree-shaped structures $[47,15,48]$. Seen at the "core language" level, WS2S is obtained from WS1S by replacing the single successor predicate by two successor predicates, for left and right successor respectively. This logic is also decidable by the automaton-logic connection, but using tree automata instead of string automata. The MonA tool also implements this decision procedure.

There is a subtle difference between WS1S, the logic now used in MonA, and M2L-Str, the logic used in early experimental versions $[48,6,16]$. (The difference between WS2S and M2L-Tree is similar.) In WS1S, formulas are interpreted over infinite string models (but quantification is restricted to finite sets only). In M2L-Str, formulas are instead interpreted over finite string models. That is, the universe is not the whole set of naturals $\mathbb{N}$, but a bounded subset $\{0, \ldots, n-1\}$, where $n$ is defined by the length of the string. The decision procedure for M2L-Str is almost the same as for WS1S, only slightly simpler: the quotient operation (before projection) is just omitted. From the language point of view, M2L-Str corresponds exactly to the regular languages (all formulas correspond to automata and vice versa), and WS1S corresponds to those regular languages that are closed under concatenation by 0's. These properties make M2L-Str preferable for some applications [4, 44]. However, the fact that not all positions have a successor often makes M2L-Str rather unnatural to use. Being closer tied to arithmetic, the WS1S semantics is easier to understand. Also, for instance Presburger Arithmetic can easily be encoded in WS1S whereas there is no obvious encoding in M2L-Str.

Notice that the most significant source of complexity in this decision procedure is the quantifiers, or more precisely, the automaton determinization. Each quantifier can cause an exponential blow-up in the number of automaton states, 
so in worst case, this decision procedure has a non-elementary complexity. Furthermore, we cannot hope for a fundamentally better decision procedure since this is also the lower bound for the WS1S decision problem [37]. However, as we will show, even constant factors of improvement can make significant differences in practice.

To make matters even worse (and the challenge the more interesting), the implementation also has to deal with automata with huge alphabets. As mentioned, if $\phi_{0}$ has $k$ free variables, the alphabet is $\mathbb{B}^{k}$. Standard automaton packages cannot handle alphabets of that size, for typical values of $k$.

\section{Benchmark Formulas}

The experiments presented in the following section are based on twelve benchmark formulas, here shown with their sizes, the logics they are using, and their time and space consumptions when processed by MoNA 1.4 (on a 296MHz UltraSPARC with 1GB RAM):

\begin{tabular}{|c|l|r|l|r|r|}
\hline Benchmark & \multicolumn{1}{|c|}{ Name } & \multicolumn{1}{c|}{ Size } & \multicolumn{1}{c|}{ Logic } & \multicolumn{1}{c|}{ Time } & \multicolumn{1}{c|}{ Space } \\
\hline \hline A & dflipflop.mona & $2 \mathrm{~KB}$ & WS1S (M2L-Str) & $0.4 \mathrm{sec}$ & $3 \mathrm{MB}$ \\
$\mathrm{B}$ & euclid.mona & $6 \mathrm{~KB}$ & WS1S (Presburger) & $33.1 \mathrm{sec}$ & $217 \mathrm{MB}$ \\
$\mathrm{C}$ & fischer_mutex.mona & $43 \mathrm{~KB}$ & WS1S & $15.1 \mathrm{sec}$ & $13 \mathrm{MB}$ \\
$\mathrm{D}$ & html3_grammar.mona & $39 \mathrm{~KB}$ & WS2S (WSRT) & $137.1 \mathrm{sec}$ & $208 \mathrm{MB}$ \\
$\mathrm{E}$ & lift_controller.mona & $36 \mathrm{~KB}$ & WS1S & $8.0 \mathrm{sec}$ & $15 \mathrm{MB}$ \\
F & mcnc91_bbsse.mona & $9 \mathrm{~KB}$ & WS1S & $13.2 \mathrm{sec}$ & $17 \mathrm{MB}$ \\
G & reverse_linear.mona & $11 \mathrm{~KB}$ & WS1S (M2L-Str) & $3.2 \mathrm{sec}$ & $4 \mathrm{MB}$ \\
$\mathrm{H}$ & search_tree.mona & $19 \mathrm{~KB}$ & WS2S (WSRT) & $30.4 \mathrm{sec}$ & $5 \mathrm{MB}$ \\
I & sliding_window.mona & $64 \mathrm{~KB}$ & WS1S & $40.3 \mathrm{sec}$ & $59 \mathrm{MB}$ \\
J & szymanski_acc.mona & $144 \mathrm{~KB}$ & WS1S & $20.6 \mathrm{sec}$ & $9 \mathrm{MB}$ \\
K & von_neumann_adder.mona & $5 \mathrm{~KB}$ & WS1S & $139.9 \mathrm{sec}$ & $116 \mathrm{MB}$ \\
L & xbar_theory.mona & $14 \mathrm{~KB}$ & WS2S & $136.4 \mathrm{sec}$ & $518 \mathrm{MB}$ \\
\hline
\end{tabular}

The benchmarks have been picked from a large variety of MoNA applications ranging from hardware verification to encoding of natural languages.

dflipflop.mona - a verification of a D-type flip-flop circuit [4]. Provided by Abdel Ayari.

euclid.mona - an encoding in Presburger arithmetic of six steps of reachability on a machine that implements Euclid's GCD algorithm [45]. Provided by Tom Shiple.

fischer_mutex.mona and lift_controller.mona - duration calculus encodings of Fischer's mutual exclusion algorithm and a mine pump controller, translated to Mona code [42]. Provided by Paritosh Pandya.

html3_grammar.mona - a tree-logic encoding of the HTML 3.0 grammar annotated with 10 parse-tree formulas [14]. Provided by Niels Damgaard. 
reverse_linear.mona - verifies correctness of a $\mathrm{C}$ program reversing a pointerlinked list [23].

search_tree.mona - verifies correctness of a $\mathrm{C}$ program deleting a node from a search tree [17].

sliding_window.mona - verifies correctness of a sliding window network protocol [46]. Provided by Mark Smith.

szymanski_acc.mona - validation of the parameterized Szymanski problem using an accelerated iterative analysis [9]. Provided by Mamoun Filali-Amine.

von_neumann_adder.mona and mcnc91_bbsse.mona - verification of sequential hardware circuits; the first verifies that an 8-bit von Neumann adder is equivalent to a standard carry-chain adder, the second is a benchmark from MCNC91 [49]. Provided by Sebastian Mödersheim.

xbar_theory.mona - encodes a part of a theory of natural languages in the Chomsky tradition. It was used to verify the theory and led to the discovery of mistakes in the original formalization [39]. Provided by Frank Morawietz.

We will use these benchmarks to illustrate the effects of the various implementation "secrets" by comparing the efficiency of MoNA shown in the table above with that obtained by handicapping the MoNA implementation by not using the techniques.

\section{Implementation Secrets}

The Mona implementation has been developed and tuned over a period of six years. Many large and small ideas have contributed to a combined speedup of several orders of magnitude. Improvements have taken place at all levels, which we illustrate with the following seven examples from different phases of the compilation and execution of formulas.

To enable comparisons, we summarize the effect of each implementation "secret" by a single dimensionless number for each benchmark formula. Usually, this is simply the speedup factor, but in some cases where the numerator is not available, we argue for a more synthetic measure. If a benchmark cannot run on our machine, it is assigned time $\infty$.

\subsection{BDD-based automata representation}

The very first attempt to implement the decision procedure used a representation based on conjunctive normal forms - however this quickly showed to be very inefficient. The first actually useful version of the MONA tool was the 1995 experimental ML-version [21]. The reason for the success was the novel representation of automata based on (reduced and ordered) BDDs (Binary Decision Diagrams) $[10,11]$ for addressing the problem of large alphabets. In addition, the representation allows some specialized algorithms to be applied [33, 27]. 
A BDD is a graph representing a boolean function. The BDD representation has some extremely convenient properties, such as compactness and canonicity, and it allows efficient manipulation. BDDs have successfully been used in a long range of verification techniques (a popular one is [36]). In MoNA, a special form of BDDs, called shared multi-terminal BDDs, or SMBDDs are used. As an example, the transition function of the tiny automaton shown in Section 2 is represented in MONA as the following SMBDD:

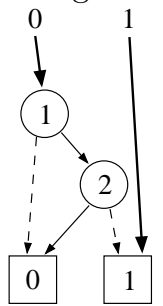

The roots and the leaves represent the states. Each root has an edge to the node representing its alphabet part of the transition function. For the other edges, dashed represents 0 and solid represents 1 . As an example, from state 0 , the transition labeled $\left(\begin{array}{l}1 \\ 0\end{array}\right)$ leads to state 1 . In this way, states are still represented explicitly, but the transitions are represented symbolically, in a compact way.

Its reasonable to ask: "What would happen if we had simply represented the transition tables in a standard fashion, that is, a row for each state and a column for each letter?". Under this point of view, it makes sense to define a letter for each bit-pattern assignment to the free variables of a subformula (as opposed to the larger set of all variables bound by an outer quantifier). We have instrumented MonA to measure the sum of the number of entries of all such automata transition tables constructed during a run of a version of MONA without BDDs:

\begin{tabular}{|c|r|r|r|}
\hline & \multicolumn{1}{|c|}{ Misses } & \multicolumn{1}{|c|}{ Table entries } & \multicolumn{1}{|c|}{ Effect } \\
\hline \hline $\mathrm{A}$ & 397,472 & 237,006 & 0.6 \\
$\mathrm{~B}$ & $48,347,395$ & $2,973,118$ & 0.1 \\
$\mathrm{C}$ & $46,080,139$ & $1,376,499,745,600$ & $29,871.9$ \\
$\mathrm{E}$ & $19,208,299$ & $290,999,305,488$ & $15,149.7$ \\
$\mathrm{~F}$ & $39,942,638$ & $2,844,513,432,416,357,974,016$ & $71,214,961,626,128.9$ \\
$\mathrm{G}$ & 561,202 & 912,194 & 1.6 \\
$\mathrm{I}$ & $95,730,831$ & $116,387,431,997,281,136$ & $1,215,777,934.7$ \\
$\mathrm{~J}$ & $24,619,563$ & $15,424,761,908$ & 626.5 \\
$\mathrm{~K}$ & $250,971,828$ & $2,544,758,557,238,438$ & $10,139,618.4$ \\
\hline
\end{tabular}

In Section 4.2, we describe the importance of cache awareness, which motivates the number of cache misses as a reasonable efficiency measure. "Misses" is the number of cache misses in our BDD-based implementation, and "Table entries" is the total number of table entries in the naive implementation. To roughly estimate the effect of the BDD-representation, we conservatively assume that each table entry results in just a single cache miss; thus, "Effect" compares "Table entries" to "Misses". The few instances where the effect is less than 
one correctly identify benchmark formulas where the BDDs are less necessary, but are also artifacts of our conservative assumption. Conversely, the extremely high effects are associated with formulas that could not possibly be decided without BDDs. Of course, the use of BDD-structures completely dominates all other optimizations, since no implementation could realistically be based on the naive table representation.

The BDD-representation was the first breakthrough of the MonA implementation, and the other "secrets" should really be viewed with this as baseline.

\subsection{Cache-conscious data structures}

The data structure used to represent the BDDs for transition functions has been carefully tuned to minimize the number of cache misses that occur. This effort is motivated in earlier work [33], where it is determined that the number of cache misses during unary and binary BDD apply steps totally dominates the running time.

In fact, we argued that if A1 is the number of unary apply steps and A2 is the number of binary apply steps, then there exists constant $m, c_{1}$, and $c_{2}$ such that the total running time is approximately $m\left(c_{1} \cdot \mathrm{A} 1+c_{2} \cdot \mathrm{A} 2\right)$. Here, $m$ is the machine dependent delay incurred by an L2 cache miss, and $c_{1}$ and $c_{2}$ are the average number of cache misses for unary and binary apply steps. This estimate is based the assumption that time incurred for manipulating auxiliary data structures, such as those used for describing subsets in the determinization construction, is insignificant. For the machine we have used for experiments, it is by a small $\mathrm{C}$ utility determined that $m=0.43 \mu \mathrm{s}$. In our BDD implementation, explained in [33], we have estimated from algorithmic considerations that $c_{1}=$ 1.7 and $c_{2}=3$ (the binary apply may entail the use of unary apply steps for doubling tables that were too small - these steps are not counted towards the time for binary apply steps, and that is why we can use the figure $c_{2}=3$ ); we also estimated that for an earlier conventional implementation, the numbers were $c_{1}=6.7$ and $c_{2}=7.3$. The main reason for this difference is that our specialized package stores nodes directly under their hash address to minimize cache misses; traditional BDD packages store BDD nodes individually with the hash table containing pointers to them-roughly doubling the time it takes to process a node. We no longer support the conventional BDD implementation, so to measure the effect of cache-consciousness, we must use the above formula to estimate the running times that would have been obtained today.

In the following experiment, we have instrumented MonA to obtain the exact numbers of apply steps: 


\begin{tabular}{|c|r|r|r|r|r|r|r|}
\hline & \multicolumn{1}{|c|}{ Apply1 } & \multicolumn{1}{c|}{ Apply2 } & \multicolumn{1}{c|}{ Misses } & \multicolumn{1}{c|}{ Auto } & Predicted & Conventional & Effect \\
\hline \hline A & 183,949 & 28,253 & 397,472 & $0.2 \mathrm{sec}$ & $0.2 \mathrm{sec}$ & $0.6 \mathrm{sec}$ & 3.0 \\
$\mathrm{~B}$ & $21,908,722$ & $3,700,856$ & $48,347,395$ & $32.8 \mathrm{sec}$ & $20.8 \mathrm{sec}$ & $74.7 \mathrm{sec}$ & 3.6 \\
$\mathrm{C}$ & $24,585,292$ & $1,428,381$ & $46,080,139$ & $14.2 \mathrm{sec}$ & $19.8 \mathrm{sec}$ & $75.2 \mathrm{sec}$ & 3.8 \\
$\mathrm{E}$ & 9,847007 & 822,796 & $19,208,299$ & $7.7 \mathrm{sec}$ & $8.2 \mathrm{sec}$ & $30.9 \mathrm{sec}$ & 3.8 \\
$\mathrm{~F}$ & $13,406,047$ & $5,717,453$ & $39,942,638$ & $12.8 \mathrm{sec}$ & $17.2 \mathrm{sec}$ & $56.6 \mathrm{sec}$ & 3.3 \\
G & 233,566 & 54,814 & 561,504 & $0.5 \mathrm{sec}$ & $0.3 \mathrm{sec}$ & $0.8 \mathrm{sec}$ & 2.7 \\
I & $36,629,195$ & $11,153,733$ & $95,730,831$ & $37.0 \mathrm{sec}$ & $41.2 \mathrm{sec}$ & $140.5 \mathrm{sec}$ & 3.4 \\
$\mathrm{~J}$ & $10,497,759$ & $2,257,791$ & $24,619,563$ & $11.6 \mathrm{sec}$ & $10.6 \mathrm{sec}$ & $37.3 \mathrm{sec}$ & 3.5 \\
K & $129,126,447$ & $10,485,623$ & $250,971,828$ & $137.4 \mathrm{sec}$ & $107.9 \mathrm{sec}$ & $404.7 \mathrm{sec}$ & 3.8 \\
\hline
\end{tabular}

"Apply1" is the number of unary apply steps; "Apply2" is the number of binary apply steps; "Misses" is the number of cache misses predicted by the formula above; "Auto" is the part of the actual running time involved in automata constructions; "Predicted" is the running time predicted from the cache misses alone; "Conventional" is the predicted running time for a conventional BDD implementation that was not cache-conscious; and "Effect" is "Conventional" compared to "Predicted". In most cases, the actual running time is close to the predicted one (within 25\%). Note that there are instances where the actual time is about $50 \%$ larger than the estimated time: benchmark B involves a lengthy subset construction on an automaton with small BDDs - thus it violates the assumption that the time handling accessory data structures is insignificant; similarly, benchmark $\mathrm{G}$ also consists of many automata with few BDD nodes prone to violating the assumption.

In an independent comparison [45] it was noted that MoNA was consistently twice as fast as a specially designed automaton package based on a BDD package considered efficient. In [33], the comparison to a traditional BDD package yielded a factor 5 speedup.

\subsection{Eager minimization}

When MonA inductively translates formulas to automata, a Myhill-Nerode minimization is performed after every product and projection operation. Naturally, it is preferable to operate with as small automata as possible, but our strategy may seem excessive since minimization often exceeds $50 \%$ of the total running time. This suspicion is strengthened by the fact that MonA automata by construction contain only reachable states; thus, minimization only collapses redundant states.

Three alternative strategies to the eager one currently used by MonA would be to perform only the very final minimization, only the ones occurring after projection operations, or only the ones occurring after product operations. Many other heuristics could of course also be considered. The following table results from such an investigation: 


\begin{tabular}{|c|c|c|c|c|c|}
\hline & \multicolumn{4}{|c|}{ Time } & \multirow{2}{*}{ Effect } \\
\hline & Only final & After project & After product & Always & \\
\hline$\overline{\mathrm{A}}$ & $\infty$ & $\infty$ & $0.6 \mathrm{sec}$ & $0.4 \mathrm{sec}$ & 1.5 \\
\hline B & $\infty$ & $\infty$ & $\infty$ & $33.1 \mathrm{sec}$ & $\infty$ \\
\hline $\mathrm{C}$ & $\infty$ & $\infty$ & $32.3 \mathrm{sec}$ & $15.1 \mathrm{sec}$ & 2.1 \\
\hline $\mathrm{D}$ & $\infty$ & $\infty$ & $290.6 \mathrm{sec}$ & $137.1 \mathrm{sec}$ & 2.1 \\
\hline $\mathrm{E}$ & $\infty$ & $\infty$ & $19.4 \mathrm{sec}$ & $8.0 \mathrm{sec}$ & 2.4 \\
\hline $\mathrm{F}$ & $\infty$ & $\infty$ & $36.7 \mathrm{sec}$ & $13.2 \mathrm{sec}$ & 2.8 \\
\hline $\mathrm{G}$ & $\infty$ & $\infty$ & $5.8 \mathrm{sec}$ & $3.2 \mathrm{sec}$ & 1.8 \\
\hline $\mathrm{H}$ & $\infty$ & $\infty$ & $59.6 \mathrm{sec}$ & $30.4 \mathrm{sec}$ & 2.0 \\
\hline I & $\infty$ & $\infty$ & $74.4 \mathrm{sec}$ & $40.3 \mathrm{sec}$ & 1.8 \\
\hline $\mathrm{J}$ & $\infty$ & $\infty$ & $36.3 \mathrm{sec}$ & $20.6 \mathrm{sec}$ & 1.8 \\
\hline K & $\infty$ & $\infty$ & $142.3 \mathrm{sec}$ & $139.9 \mathrm{sec}$ & 1.0 \\
\hline $\mathrm{L}$ & $\infty$ & $\infty$ & $\infty$ & $136.4 \mathrm{sec}$ & $\infty$ \\
\hline
\end{tabular}

"Only final" is the running time when minimization is only performed as the final step of the translation; "After project" is the running time when minimization is also performed after every projection operation; "After product" is the running time when minimization is instead performed after every product operation; "Always" is the time when minimization is performed eagerly; and "Effect" is the "After product" time compared to the "Always" time (since the other two strategies are clearly hopeless). Eager minimization is seen to be always beneficial and in some cases essential for the benchmark formulas.

\subsection{Guided tree automata}

Tree automata are inherently more computationally expensive because of their three-dimensional transition tables. We have used a technique of factorization of state spaces to split big tree automata into smaller ones. The basic idea, which may result in exponential savings, is explained in [7, 30]. To exploit this feature, the MonA programmer must manually specify a guide, which is a topdown tree automaton that assigns state spaces to the nodes of a tree. However, when using the WSRT logic, a canonical guide is automatically generated. For our two WSRT benchmarks, we measure the effect of this canonical guide:

\begin{tabular}{|l|r|r|r|}
\hline & Without guide & With guide & Effect \\
\hline \hline $\mathrm{D}$ & $584.0 \mathrm{sec}$ & $137.1 \mathrm{sec}$ & 4.3 \\
$\mathrm{H}$ & $\infty$ & $30.4 \mathrm{sec}$ & $\infty$ \\
\hline
\end{tabular}

"Without guide" shows the running time without any guide, while "With guide" shows the running time with the canonical WSRT guide; "Effect" shows the "Without guide" time compared to the "With guide" time. We have only a small sample space here, but clearly guides are very useful. This is hardly surprising, since they may yield an asymptotic improvement in running time. 


\subsection{DAGification}

Internally, MonA is divided into a front-end and a back-end. The front-end parses the input and builds a data structure representing the automata-theoretic operations that will calculate the resulting automaton. The back-end then inductively carries out these operations.

The generated data structure is often seen to contain many common subformulas. This is particularly true when they are compared relative to signature equivalence, which holds for two formulas $\phi$ and $\phi^{\prime}$ if there is an order-preserving renaming of the variables in $\phi$ (increasing with respect to the indices of the variables) such that the representations of $\phi$ and $\phi^{\prime}$ become identical.

A property of the BDD representation is that the automata corresponding to signature-equivalent trees are isomorphic in the sense that only the node indices differ. This means that intermediate results can be reused by simple exchanges of node indices. For this reason, MonA represents the formulas in a DAG (Directed Acyclic Graph), not a tree. The DAG is conceptually constructed from the tree using a bottom-up collapsing process, based on the signature equivalence relation as described in [16].

Clearly, constructing the DAG instead of the tree incurs some overhead, but the following experiments show that the benefits are significantly larger:

\begin{tabular}{|r||r|r||r|r||r|}
\hline \multicolumn{1}{|c||}{} & \multicolumn{2}{c||}{ Nodes } & \multicolumn{2}{c|}{ Time } & \multirow{2}{*}{ Effect } \\
\cline { 2 - 5 } & \multicolumn{1}{|c||}{ Tree } & DAG & \multicolumn{1}{c|}{ Tree } & \multicolumn{1}{c|}{ DAG } & \\
\hline \hline $\mathrm{A}$ & 2,532 & 296 & $1.7 \mathrm{sec}$ & $0.4 \mathrm{sec}$ & 4.3 \\
$\mathrm{~B}$ & 873 & 259 & $79.2 \mathrm{sec}$ & $33.1 \mathrm{sec}$ & 2.4 \\
$\mathrm{C}$ & 5,432 & 461 & $40.1 \mathrm{sec}$ & $15.1 \mathrm{sec}$ & 2.7 \\
$\mathrm{D}$ & 3,038 & 270 & $\infty$ & $137.1 \mathrm{sec}$ & $\infty$ \\
$\mathrm{E}$ & 4,560 & 505 & $20.5 \mathrm{sec}$ & $8.0 \mathrm{sec}$ & 2.6 \\
$\mathrm{~F}$ & 1,997 & 505 & $49.1 \mathrm{sec}$ & $13.2 \mathrm{sec}$ & 3.7 \\
$\mathrm{G}$ & 56,932 & 1,199 & $\infty$ & $3.2 \mathrm{sec}$ & $\infty$ \\
$\mathrm{H}$ & 8,180 & 743 & $\infty$ & $30.4 \mathrm{sec}$ & $\infty$ \\
$\mathrm{I}$ & 14,058 & 1,396 & $107.1 \mathrm{sec}$ & $40.3 \mathrm{sec}$ & 2.7 \\
$\mathrm{~J}$ & 278,116 & 6,314 & $\infty$ & $20.6 \mathrm{sec}$ & $\infty$ \\
$\mathrm{K}$ & 777 & 273 & $284.0 \mathrm{sec}$ & $139.9 \mathrm{sec}$ & 2.0 \\
$\mathrm{~L}$ & 1,504 & 388 & $\infty$ & $136.4 \mathrm{sec}$ & $\infty$ \\
\hline
\end{tabular}

"Nodes" shows the number of nodes in the representation of the formula. "Tree" is the number of nodes using an explicit tree representation, while "DAG" is the number of nodes after DAGification. "Time" shows the running times for the same two cases. "Effect" shows the "Tree" running time compared to the "DAG" running time. From the differences in the number of nodes, one might expect the total effect to be larger, however DAGification is mainly effective on small formulas where the corresponding automata typically are also smaller. Nevertheless, the DAGification process is seen to provide a substantial and often essential gain in efficiency.

The effects reported sometimes benefit from the fact that the restriction technique presented in the following subsection knowingly generates redundant 
formulas. This explains some of the failures observed.

\subsection{Three-valued logic and automata}

The standard technique for dealing with first-order variables in monadic secondorder logics is to encode them as second-order variables, typically as singletons. However, that raises the issue of restrictions: the common phenomenon that a formula $\phi$ makes sense, relative to some exterior conditions, only when an associated restriction holds. The restriction is also a formula, and the main issue is that $\phi$ is now essentially undefined outside the restriction. In the case of first-order variables encoded as second-order variables, the restriction is that these variables are singletons. We experienced the same situation trying to emulate M2L-Str in WS1S, causing state-space explosions.

The nature of these problems is technical, but fortunately they can be solved through a theory of restriction couched in a three-valued logic [28]. Under this view, a restricted subformula $\phi$ is associated with a restriction $\phi_{R}$. If, for some valuation, $\phi_{R}$ does not hold, then formulas containing $\phi$ are assigned a new third truth value "don't-care". This three-valued logic carries over to the MonA notion of automata - in addition to accept and reject states, they also have "don't-care" states. A special restrict $\left(\phi_{R}\right)$ operation is used for converting reject states to "don't-care" states for the restriction formulas, and the other automaton operations are modified to ensure that non-acceptance of restrictions is propagated properly.

This gives a cleaner semantics to the restriction phenomenon, and furthermore avoids the state-space explosions mentioned above. According to [28], we can guarantee that the WS1S framework handles all formulas written in M2LStr, even with intermediate automata that are no bigger than when using the traditional M2L-Str decision procedure. MonA uses the same technique for the tree logics, WS2S and M2L-Tree.

We refer to [28] for the full theory of three-valued logic and automata. Unfortunately, there is no way of disabling this feature to provide a quantitative comparison.

\subsection{Formula reductions}

Formula reduction is a means of "optimizing" the formulas in the DAG before translating them into automata. The reductions are based on a syntactic analysis that attempts to identify valid subformulas and equivalences among subformulas.

There are some non-obvious choices here. How should computation resources be apportioned to the reduction phase and to the automata calculation phase? Must reductions guarantee that automata calculations become faster? Should the two phases interact? Our answers are based on some trial and error along with some provisions to cope with subtle interactions with other of our optimization secrets. 
Mona 1.4 performs three kinds of formula reductions: 1) simple equality and boolean reductions, 2) special quantifier reductions, and 3) special conjunction reductions. The first kind can be described by simple rewrite rules (only some typical ones are shown):

$$
\begin{array}{rlll}
X_{i}=X_{i} & \rightsquigarrow \text { true } & \phi \& \phi & \rightsquigarrow \phi \\
\text { true \& } \phi & \rightsquigarrow \phi & \sim \phi & \rightsquigarrow \phi \\
\text { false \& } \phi & \rightsquigarrow \text { false } & \sim \text { false } & \rightsquigarrow \text { true }
\end{array}
$$

These rewrite steps are guaranteed to reduce complexity, but will not cause significant improvements in running time, since they all either deal with constant size automata or rarely apply in realistic situations. Nevertheless, they are extremely cheap, and they may yield small improvements, in particular on machine generated MonA code.

The second kind of reductions can potentially cause tremendous improvements. As mentioned, the non-elementary complexity of the decision procedure is caused by the automaton projection operations, which stem from quantifiers. The accompanying determinization construction may cause an exponential blow-up in automaton size. Our basic idea is to apply a rewrite step resembling let-reduction, which removes quantifiers:

$$
\begin{aligned}
& \text { ex2 } X_{i}: \phi \rightsquigarrow \phi\left[T / X_{i}\right] \quad \begin{array}{l}
\text { provided that } \phi=>X_{i}=T \text { is valid, } \\
\text { and } T \text { is some term satisfying } F V(T) \subseteq
\end{array} \\
& F V(\phi)
\end{aligned}
$$

where $F V(\cdot)$ denotes the set of free variables. For several reasons, this is not the way to proceed in practice. First of all, finding terms $T$ satisfying the side condition can be an expensive task, in worst case non-elementary. Secondly, the translation into automata requires the formulas to be "flattened" by introduction of quantifiers such that there are no nested terms. So, if the substitution $\phi[T / X]$ generates nested terms, then the removed quantifier is recreated by the translation. Thirdly, when the rewrite rule applies in practice, $\phi$ usually has a particular structure as reflected in the following more restrictive rewrite rule chosen in Mona:

$$
\begin{aligned}
\text { ex2 } X_{i}: \phi \rightsquigarrow \phi\left[X_{j} / X_{i}\right] & \text { provided that } \phi \\
& \cdots \& \& X_{i}=X_{j} \& \quad \cdots \text { and } X_{j} \\
& \text { is some variable other than } X_{i}
\end{aligned}
$$

In contrast to equality and boolean reductions, this rule is not guaranteed to improve performance, since substitutions may cause the DAG reuse degree to decrease.

The third kind of reductions applies to conjunctions, of which there are two special sources. One is the formula flattening just mentioned; the other is the formula restriction technique mentioned in Section 4.6. Both typically introduce many new conjunctions. Studies of a graphical representation of the formula DAGs (MonA can create such graphs automatically) led us to believe that many 
of these new conjunctions are redundant. A typical rewrite rule addressing such redundant conjunctions is the following:

$$
\begin{aligned}
\phi_{1} \& \phi_{2} \rightsquigarrow \phi_{1} & \text { provided that nonrestr }\left(\phi_{2}\right) \subseteq \text { nonrestr }\left(\phi_{1}\right) \cup \\
& \operatorname{restr}\left(\phi_{1}\right) \text { and } \operatorname{restr}\left(\phi_{2}\right) \subseteq \operatorname{restr}\left(\phi_{1}\right)
\end{aligned}
$$

Here, $\operatorname{restr}(\phi)$ is the set of restrict (.) conjuncts in $\phi$, and nonrestr $(\phi)$ is the set of non-restrict $(\cdot)$ conjuncts in $\phi$. This reduction states that it is sufficient to assert $\phi_{1}$ when $\phi_{1} \& \phi_{2}$ was originally asserted in situations where the nonrestricted conjuncts of $\phi_{2}$ are already conjuncts of $\phi_{1}$-whether restricted or not - and the restricted conjuncts of $\phi_{2}$ are non-restricted conjuncts of $\phi_{1}$. It is not sufficient that they be restricted conjuncts of $\phi_{1}$, since the restrictions may not be the same in $\phi_{1}$.

All rewrite rules mentioned here have the property that they cannot "do any harm", that is, have a negative impact on the automaton sizes. (They can however damage the reuse factor obtained by the DAGification, but this is rarely a problem in practice.) A different kind of rewrite rules could be obtained using heuristic - this will be investigated in the future.

With the DAG representation of formulas, the reductions just described can be implemented relatively easily in MonA. The table below shows the effects

\begin{tabular}{|c|c|c|c|c|c|c|c|c|c|}
\hline & \multicolumn{3}{|c|}{ Hits } & \multicolumn{5}{|c|}{ Time } & \multirow{2}{*}{ Effect } \\
\hline & Simple & Quant. & Conj. & None & Simple & Quant. & Conj. & All & \\
\hline$\overline{\mathrm{A}}$ & 12 & 8 & 22 & $0.8 \mathrm{sec}$ & $0.7 \mathrm{sec}$ & $0.7 \mathrm{sec}$ & $0.7 \mathrm{sec}$ & $0.4 \mathrm{sec}$ & 2.0 \\
\hline B & 10 & 45 & 0 & $58.2 \mathrm{sec}$ & $58.8 \mathrm{sec}$ & $56.2 \mathrm{sec}$ & $56.8 \mathrm{sec}$ & $33.1 \mathrm{sec}$ & 1.8 \\
\hline $\mathrm{C}$ & 9 & 13 & 8 & $43.7 \mathrm{sec}$ & $41.9 \mathrm{sec}$ & $37.1 \mathrm{sec}$ & $42.9 \mathrm{sec}$ & $15.1 \mathrm{sec}$ & 2.9 \\
\hline $\mathrm{D}$ & 4 & 28 & 27 & $542.7 \mathrm{sec}$ & $536.1 \mathrm{sec}$ & $296.0 \mathrm{sec}$ & $404.7 \mathrm{sec}$ & $137.1 \mathrm{sec}$ & 4.0 \\
\hline $\mathrm{E}$ & 5 & 6 & 19 & $22.6 \mathrm{sec}$ & $23.4 \mathrm{sec}$ & $16.6 \mathrm{sec}$ & $22.7 \mathrm{sec}$ & $8.0 \mathrm{sec}$ & 2.8 \\
\hline $\mathrm{F}$ & 3 & 1 & 1 & $28.3 \mathrm{sec}$ & $29.9 \mathrm{sec}$ & $27.0 \mathrm{sec}$ & $27.2 \mathrm{sec}$ & $13.2 \mathrm{sec}$ & 2.1 \\
\hline G & 65 & 318 & 191 & $6.1 \mathrm{sec}$ & $5.9 \mathrm{sec}$ & $6.1 \mathrm{sec}$ & $5.9 \mathrm{sec}$ & $3.2 \mathrm{sec}$ & 1.9 \\
\hline $\mathrm{H}$ & 35 & 32 & 81 & $104.1 \mathrm{sec}$ & $102.6 \mathrm{sec}$ & $71.0 \mathrm{sec}$ & $98.5 \mathrm{sec}$ & $30.4 \mathrm{sec}$ & 3.4 \\
\hline I & 102 & 218 & 7 & $76.2 \mathrm{sec}$ & $76.5 \mathrm{sec}$ & $75.0 \mathrm{sec}$ & $76.0 \mathrm{sec}$ & $40.3 \mathrm{sec}$ & 1.9 \\
\hline $\mathrm{J}$ & 91 & 0 & 1 & $37.3 \mathrm{sec}$ & $37.9 \mathrm{sec}$ & $37.6 \mathrm{sec}$ & $37.0 \mathrm{sec}$ & $20.6 \mathrm{sec}$ & 1.9 \\
\hline K & 9 & 4 & 1 & $313.7 \mathrm{sec}$ & $267.9 \mathrm{sec}$ & $240.3 \mathrm{sec}$ & $302.6 \mathrm{sec}$ & $139.9 \mathrm{sec}$ & 2.3 \\
\hline $\mathrm{L}$ & 4 & 4 & 18 & $\infty$ & $\infty$ & $\infty$ & $\infty$ & $136.4 \mathrm{sec}$ & $\infty$ \\
\hline
\end{tabular}
of performing the reductions on the benchmark formulas:

"Hits" shows the number of times each of the three kinds of reduction is performed; "Time" shows the total running time in the cases where no reductions are performed, only the first kind of reductions are performed, only the second, only the third, and all of them together. "Effect" shows the "None" times compared to the "All" times. All benchmarks gain from formula reductions, and in a single example this technique is even necessary. Note that most often all three kinds of reductions must act in unison to obtain significant effects.

A general benefit from formula reductions is that tools generating MoNA formulas from other formalisms may generate naive and voluminous output 
while leaving optimizations to MonA. In particular, tools may use existential quantifiers to bind terms to fresh variables, knowing that MONA will take care of the required optimization.

\section{$5 \quad$ Future Developments}

Several of the techniques described in the previous section can be further refined of course. The most promising ideas seem however to concentrate on the BDD representation. In the following, we describe three such ideas.

It is a well-known fact [10] that the ordering of variables in the BDD automata representation has a strong influence on the number of BDD nodes required. The impact of choosing a good ordering can be an exponential improvement in running times. Finding the optimal ordering is an NP-complete problem, but we plan to experiment with the heuristics that have been suggested [13].

We have sometimes been asked: "Why don't you encode the states of the automata in BDDs, since that is a central technique in model checking?". The reason is that there is no obvious structure to the state space in most cases that would lend itself towards an efficient BDD representation. For example, consider the consequences of a subset construction or a minimization construction, where similar states are collapsed; in either case, it is not obvious how to represent the new state. However, the ideas are worth investigating.

For our tree automata, we have experimentally observed that the use of guides produce a large number of component automata many of which are almost identical. We will study how to compress this representation using a BDD-like global structure.

\section{Conclusion}

The presented techniques reflect a lengthy Darwinian development process of the Mona tool in which only robust and useful ideas have survived. We have not mentioned here the many ideas that failed or were surpassed by other techniques. Our experiences confirm the maxim that optimizations must be carried out at all levels and that no single silver bullet is sufficient. We are confident that further improvements are still possible and that many other interesting applications will be made.

\section{Acknowledgments}

Many people have contributed to the decelopment of MONA, in particular we are grateful to David Basin, Morten Biehl, Jacob Elgaard, Jesper Gulmann, Jacob Jensen, Michael Jørgensen, Bob Paige, Theis Rauhe, and Anders Sandholm. We also thank the MONA users who kindly provided the benchmark formulas. 


\section{References}

[1] Abdelwaheb Ayari, David Basin, and Stefan Friedrich. Structural and behavioral modeling with monadic logics. In The Twenty-Ninth IEEE International Symposium on Multiple-Valued Logic. IEEE Computer Society, 1999.

[2] Abdelwaheb Ayari, David Basin, and Andreas Podelski. LISA: A specification language based on WS2S. In CSL '97, LNCS, 1998.

[3] David Basin and Stefan Friedrich. Combining WS1S and HOL. In Frontiers of Combining Systems 2, volume 7 of Studies in Logic and Computation. Research Studies Press/Wiley, 2000.

[4] David Basin and Nils Klarlund. Automata based symbolic reasoning in hardware verification. Formal Methods In System Design, 13:255-288, 1998. Extended version of: "Hardware verification using monadic second-order logic," CAV' '95, LNCS 939.

[5] Kai Baukus, Karsten Stahl, Saddek Bensalem, and Yassine Lakhnech. Abstracting WS1S systems to verify parameterized networks. In Proc. TACAS 2000, 6th International Conference on Tools and Algorithms for the Construction and Analysis of Systems, volume 1785 of LNCS, 2000.

[6] Morten Biehl, Nils Klarlund, and Theis Rauhe. Mona: decidable arithmetic in practice (demo). In Formal Techniques in Real-Time and Fault-Tolerant Systems, 4th International Symposium, volume 1135 of LNCS, 1996.

[7] Morten Biehl, Nils Klarlund, and Theis Rauhe. Algorithms for guided tree automata. In First International Workshop on Implementing Automata, WIA '96, volume 1260 of LNCS, 1997.

[8] Jean-Paul Bodeveix and Mamoun Filali. Quantifier elimination technics for program validation. Technical report, IRIT 97-44-R, 1997.

[9] Jean-Paul Bodeveix and Mamoun Filali. FMona: a tool for expressing validation techniques over infinite state systems. In Proc. TACAS 2000, 6th International Conference on Tools and Algorithms for the Construction and Analysis of Systems, volume 1785 of LNCS, 2000.

[10] R.E. Bryant. Graph-based algorithms for boolean function manipulation. IEEE Transactions on Computers, C-35(8):677-691, Aug 1986.

[11] R.E. Bryant. Symbolic boolean manipulation with ordered binary-decision diagrams. ACM Computing surveys, 24(3):293-318, September 1992.

[12] J.R. Büchi. Weak second-order arithmetic and finite automata. Z. Math. Logik Grundl. Math., 6:66-92, 1960. 
[13] K.M. Butler, D.E. Ross, R. Kapur, and M.R. Mercer. Heuristics to compute variable orderings for efficient manipulation of ordered binary decision diagrams. In Proc. ACM/IEEE Design Automation Conference (DAC), 1991.

[14] Niels Damgaard, Nils Klarlund, and Michael I. Schwartzbach. YakYak: Parsing with logical side constraints. In Proceedings of DLT'99, 1999.

[15] J. Doner. Tree acceptors and some of their applications. J. Comput. System Sci., 4:406-451, 1970.

[16] Jacob Elgaard, Nils Klarlund, and Anders Møller. Mona 1.x: new techniques for WS1S and WS2S. In Computer Aided Verification, CAV '98, volume 1427 of $L N C S, 1998$.

[17] Jacob Elgaard, Anders Møller, and Michael I. Schwartzbach. Compiletime debugging of $\mathrm{C}$ programs working on trees. In Proceedings of European Symposium on Programming Languages and Systems, volume 1782 of LNCS, 2000.

[18] C.C. Elgot. Decision problems of finite automata design and related arithmetics. Transactions of the American Mathematical Society, 98:21-52, 1961.

[19] J. Glenn and W. Gasarch. Implementing WS1S via finite automata. In Automata Implementation, WIA '96, volume 1260 of LNCS, 1997.

[20] Aarti Gupta and Allan L. Fisher. Representation and symbolic manipulation of linearly inductive boolean functions. In Proceedings of the IEEE International Conference on Computer-Aided Design. IEEE Computer Society Press, 1993.

[21] J.G. Henriksen, J. Jensen, M. Jørgensen, N. Klarlund, B. Paige, T. Rauhe, and A. Sandholm. Mona: Monadic second-order logic in practice. In Tools and Algorithms for the Construction and Analysis of Systems, First International Workshop, TACAS '95, volume 1019 of LNCS, 1996.

[22] Thomas Hune and Anders Sandholm. A case study on using automata in control synthesis. In Proceedings of FASE'00, volume 1783 of LNCS, 2000.

[23] Jacob L. Jensen, Michael E. Jørgensen, Nils Klarlund, and Michael I. Schwartzbach. Automatic verification of pointer programs using monadic second-order logic. In PLDI '97, 1997.

[24] Bengt Jonsson and Marcus Nilsson. Transitive closures of regular relations for verifying infinite-state systems. In Proc. TACAS 2000, 6th International Conference on Tools and Algorithms for the Construction and Analysis of Systems, volume 1785 of LNCS, 2000. 
[25] P. Kelb, T. Margaria, M. Mendler, and C. Gsottberger. MOSEL: a flexible toolset for Monadic Second-order Logic. In Tools and Algorithms for Construction and Analysis of Systems, TACAS '97, volume 1217 of LNCS, 1997.

[26] Nils Klarlund. Mona \& Fido: The logic-automaton connection in practice. In Computer Science Logic, CSL '97, volume 1414 of LNCS, 1998.

[27] Nils Klarlund. An $n \log n$ algorithm for online BDD refinement. Journal of Algorithms, 32:133-154, 1999. Abbreviated version in: "Computer Aided Verification," CAV '97, LNCS 1254.

[28] Nils Klarlund. A theory of restrictions for logics and automata. In Computer Aided Verification, CAV '99, volume 1633 of LNCS, 1999.

[29] Nils Klarlund, Jari Koistinen, and Michael I. Schwartzbach. Formal design constraints. In Proceedings of OOPSLA '96, 1996.

[30] Nils Klarlund and Anders Møller. MONA Version 1.3 User Manual. BRICS Notes Series NS-98-3 (2.revision), Department of Computer Science, University of Aarhus, October 1998.

[31] Nils Klarlund, Mogens Nielsen, and Kim Sunesen. Automated logical verification based on trace abstraction. In Proceedings of PODC '96, 1996.

[32] Nils Klarlund, Mogens Nielsen, and Kim Sunesen. A case study in automated verification based on trace abstractions. In M. Broy, S. Merz, and K. Spies, editors, Formal System Specification, The RPC-Memory Specification Case Study, volume 1169 of LNCS, pages 341-374. Springer Verlag, 1996.

[33] Nils Klarlund and Theis Rauhe. BDD algorithms and cache misses. Technical report, BRICS Report Series RS-96-5, Department of Computer Science, University of Aarhus, 1996.

[34] Nils Klarlund and Michael I. Schwartzbach. A domain-specific language for regular sets of strings and trees. IEEE Transactions On Software Engineering, 25(3):378-386, 1999.

[35] O. Matz, A. Miller, A. Potthoff, W. Thomas, and E. Valkema. Report on the program AMoRE. Technical report, Report 9507, Inst. für Informatik u. Prakt. Mathematik, CAU Kiel, 1995.

[36] Ken McMillan. Symbolic Model Checking. Kluwer, 1993.

[37] A.R. Meyer. Weak monadic second-order theory of successor is not elementary recursive. In R. Parikh, editor, Logic Colloquium, (Proc. Symposium on Logic, Boston, 1972), volume 453 of LNCS, pages 132-154, 1975.

[38] Anders Møller. MONA project home page. http://www.brics.dk/mona/. 
[39] Frank Morawietz and Tom Cornell. The logic-automaton connection in linguistics. In Proceedings of LACL 1997, number 1582 in LNAI, 1997.

[40] Marcus Nilsson. Analyzing parameterized distributed algorithms. Master's thesis, Department of Computer Systems at Uppsala University, Sweden, 1999 .

[41] Sam Owre and Harald Ruess. Integrating WS1S with PVS. In Conference on Computer-Aided Verification, CAV 2000, LNCS, 2000.

[42] Paritosh K. Pandya. DCVALID 1.3: The user manual. Technical report, Tata Institute of Fundamental Research, STCS-99/1, 1999.

[43] Anders Steen Rasmussen. Symbolic model checking using monadic second order logic as requirement language. Master's thesis, Technical University of Denmark (DTU), 1999. IT-E 821.

[44] Anders Sandholm and Michael I. Schwartzbach. Distributed safety controllers for Web services. In Fundamental Approaches to Software Engineering, FASE'98, volume 1382 of LNCS, 1998.

[45] Thomas R. Shiple, James H. Kukula, and Rajeev K. Ranjan. A comparison of Presburger engines for EFSM reachability. In Computer Aided Verification, CAV '98, volume 1427 of LNCS, 1998.

[46] Mark A. Smith and Nils Klarlund. Verification of a sliding window protocol using IOA and Mona, 1999. Submitted for publication.

[47] J.W. Thatcher and J.B. Wright. Generalized finite automata with an application to a decision problem of second-order logic. Math. Systems Theory, 2:57-82, 1968.

[48] Wolfgang Thomas. Automata on infinite objects. In J. van Leeuwen, editor, Handbook of Theoretical Computer Science, volume B, pages 133-191. MIT Press/Elsevier, 1990.

[49] S. Yang. Logic synthesis and optimization benchmarks user guide version 3.0. In Tech. Rep. MCNC, 1991. 


\section{Recent BRICS Report Series Publications}

RS-00-40 Nils Klarlund, Anders Møller, and Michael I. Schwartzbach. MONA Implementation Secrets. December 2000. 19 pp. Shorter version appears in Daley, Eramian and Yu, editors, Fifth International Conference on Implementation and Application of Automata, CIAA '00 Pre-Proceedings, 2000, pages 93-102.

RS-00-39 Anders Møller and Michael I. Schwartzbach. The Pointer Assertion Logic Engine. December 2000. 23 pp. To appear in ACM SIGPLAN Conference on Programming Language Design and Implementation, PLDI '01 Proceedings, 2001.

RS-00-38 Bertrand Jeannet. Dynamic Partitioning in Linear Relation Analysis: Application to the Verification of Synchronous Programs. December 2000.

RS-00-37 Thomas S. Hune, Kim G. Larsen, and Paul Pettersson. Guided Synthesis of Control Programs for a Batch Plant using UPPAAL. December 2000. 29 pp. Appears in Hsiung, editor, International Workshop in Distributed Systems Validation and Verification. Held in conjunction with 20th IEEE International Conference on Distributed Computing Systems (ICDCS '2000), DSVV'00 Proceedings, 2000.

RS-00-36 Rasmus Pagh. Dispersing Hash Functions. December 2000. $18 \mathrm{pp}$. Preliminary version appeared in Rolim, editor, 4th. International Workshop on Randomization and Approximation Techniques in Computer Science, RANDOM '00, Proceedings in Informatics 8, 2000, pages 53-67.

RS-00-35 Olivier Danvy and Lasse R. Nielsen. CPS Transformation of Beta-Redexes. December 2000. 12 pp.

RS-00-34 Olivier Danvy and Morten Rhiger. A Simple Take on Typed Abstract Syntax in Haskell-like Languages. December 2000. 25 pp. To appear in Fifth International Symposium on Functional and Logic Programming, FLOPS '01 Proceedings, LNCS, 2001.

RS-00-33 Olivier Danvy and Lasse R. Nielsen. A Higher-Order Colon Translation. December 2000. 17 pp. To appear in Fifth International Symposium on Functional and Logic Programming, FLOPS ’01 Proceedings, LNCS, 2001. 